\title{
Identification of CpG Sites of SERPINA5 Promoter with Opposite Methylation Patterns in Benign and Malignant Prostate Cells
}

\author{
ALBERT HAGELGANS, CARSTEN JANDECK, MARKUS FRIEDEMANN, \\ ANASTASIA DONCHIN, SUSAN RICHTER and MARIO MENSCHIKOWSKI
}

Institute of Clinical Chemistry and Laboratory Medicine, Medical Faculty Carl Gustav Carus, Dresden, Germany

\begin{abstract}
Background/Aim: To date there has been no investigation into the epigenetic regulation of the serine protease inhibitor SERPINA5 in prostate cancer, where lack of this gene was considered to facilitate invasive growth patterns. Materials and Methods: Methylation degrees of eight CpG sites of SERPINA5 were analyzed in normal and malignant prostate cells using nucleotide sequencing, methylation-specific high resolution melting and digital droplet PCR techniques. Results: The methylation degree of five CPG sites significantly correlated with lower SERPINA5 expression levels. In contrast, two CpG sites (at -19 bp and $-14 \mathrm{bp}$ from the transcription start site) were hypermethylated in normal epithelial prostate cells, benign hyperplasic cells and low-invasive malignant LNCaP cells, whereas in aggressive DU-145 and PC-3 cell lines, these sites were essentially unmethylated. Conclusion: Novel methylation patterns of two distinct $C p G$ sites of the SERPINA5 promoter may be useful for differentiating benign from malignant prostate disease.
\end{abstract}

SERPINA5 is a member of the superfamily of serine protease inhibitors, which includes, amongst others, alpha1-antichymotrypsin, alpha-1-antitrypsin, antithrombin and angiotensinogen. SERPINA5 has a broader specificity than other serpins, and acts as a multi-functional protease inhibitor in different physiological processes and pathways (1-3). It is a procoagulant due to inhibition of the anticoagulant activated protein $\mathrm{C}$ and the generation of

Correspondence to: Mario Menschikowski, Institute of Clinical Chemistry and Laboratory Medicine, Medical Faculty Carl Gustav Carus, Fetscherstrasse 74, D 01307 Dresden, Germany. Tel: +49 3514582634, Fax: +49 3514584332, e-mail: Mario.Menschikowski@ uniklinikum-dresden.de

Key Words: SERPINA5, protein C inhibitor, DNA methylation, prostate cancer cells, benign prostatic hyperplasia. activated protein $\mathrm{C}$ via the thrombin-thrombomodulin complex $(1,4)$. SERPINA5 inhibits tissue- and urinary-type plasminogen activator, prostate-specific antigen and kallikrein activities, and it controls sperm motility and fertilization $(1,5)$. Human plasma SERPINA5 originates mainly from the liver (6), but it is also produced in the kidneys and reproductive organs, including testes, seminal vesicles and ovaries (1). In rodents, this protease inhibitor is present only in the reproductive organs (1). Studies using transgenic mice expressing the human SERPINA5 demonstrated its involvement in the regulation of lung remodelling, tissue regeneration, vascular permeability and proteolysis in the kidneys (1).

Importantly, SERPINA5 exerted anticancer activity preventing metastasis and angiogenesis in tumor cells and hence is considered to be a putative tumor-suppressor gene in different studies $(1,7,8)$. In breast cancer cells, SERPINA5 was found to regulate tumor cell migration, in part by inhibiting cathepsin L (9). On the other hand, SERPINA5 limited cell migration by direct interaction with fibronectin and disruption of the fibronectin-integrin signalling pathway, as shown in hepatocellular carcinoma (10). Down-regulation of SERPINA5 was demonstrated in serous ovarian carcinomas, renal and breast cancers $(8,11$ 14). In breast cancer, elevated levels of SERPINA5 correlated with increased survival and thus were considered as a positive prognostic factor in this disease (15). A potential mechanism for the contribution of SERPINA5 to a positive long-term outcome may be the suppression of tumor invasion through protease inhibition in the stroma (16).

At present, it remains unclear how the SERPINA5 level is regulated in cancer cells. One of the key mechanisms limiting the expression of different tumor-suppressor genes involved in cell proliferation and apoptosis is the tumorspecific methylation of cytosines in promoter regions (17, 18). It is noted that the development of robust and precise methods for detection of DNA methylation suitable for clinical laboratories will be of high importance $(19,20)$. 
Epigenetic regulation via $\mathrm{CpG}$ methylation is involved in the decrease of SERPINA5 levels in papillary thyroid carcinoma and in hormone receptors negative $\left(\mathrm{HR}^{-}\right)$basallike or p53 mutant breast cancers $(14,21)$. Histone modification, as another important epigenetic mechanism, also seems to be involved in the regulation of SERPINA5 expression in hepatocytes and astrocytes (22). So far there has been no investigation into a similar epigenetic regulation of SERPINA5 in prostate and other cancers. SERPINA5 was immunolocalised in tissue microarray spots to benign prostatic hyperplastic epithelium (BPH), prostatic intraepithelial neoplasia (PIN) lesions, tumours, metastases, and in all analysed prostate cancer cell lines (23). It was suggested that the lack of SERPINA5 expression in a subpopulation of high-grade tumor cells may facilitate invasive growth patterns (23). The tumor-suppressive role of SERPINA5 remains, however, questionable $(3,8,24)$. In contrast to thyroid carcinoma and breast cancer $(14,19)$, possible contributions of epigenetic mechanisms to the differential expression of SERPINA5 in prostate cancer cells is unclear.

The aim of this study was to evaluate the degree of methylation of eight $\mathrm{CpG}$ sites located in the proximal promoter of the SERPINA5 gene in normal and malignant prostate cells. To determine the $\mathrm{CpG}$ sites best discriminating between normal, benign hyperplastic and malignant cells, methylation was quantified with three different techniques, direct DNA sequencing, methylation-specific high resolution melting (MS-HRM) and droplet digital PCR (ddPCR).

\section{Materials and Methods}

Cell culture. Normal human prostate epithelial cells, PrEC (Cambrex Bio Science, Walkersville, MD, USA) and malignant prostate cell lines, PC-3, DU-145, and LNCaP (DSMZ, German Collection of Microorganisms and Cell Cultures, Braunschweig, Germany) were cultured as previously described (25). Benign prostatic hyperplastic epithelial cell line BPH-1 (DSMZ) was cultured in RPMI-1640 medium (Invitrogen, Darmstadt, Germany) supplemented with $20 \%$ fetal bovine serum (FBS; Lonza, Köln, Germany), $20 \mathrm{ng} / \mathrm{ml}$ testosterone, $5 \mu \mathrm{g} / \mathrm{ml}$ transferrin, $5 \mathrm{ng} / \mathrm{ml}$ sodium selenite, $5 \mu \mathrm{g} / \mathrm{ml}$ insulin and antibiotics.

Human mammary epithelial cells (HMEC) were purchased from Lonza and cultured in MEGM mammary epithelial cell growth medium with supplement (Lonza, Köln, Germany). Human mammary cancer cell lines, MCF-7, T-47D, SK-BR-3, Cal-51, BT474, MDA-MB-453, and MDA-MB-231 were obtained from DSMZ. MDA-MB-231 cells were cultured in DMEM, the other cell lines in RPMI 1640 culture medium (Invitrogen, Darmstadt, Germany) at $37^{\circ} \mathrm{C}$ in a humidified atmosphere of $5 \% \mathrm{CO}_{2}$. All media were supplemented with $10 \%$ FBS (Lonza), $1 \%$ penicillin/ streptomycin (Invitrogen), and $0.36 \%$ gentamycin (Invitrogen).

RNA extraction and RT-quantitative PCR analysis. RNA was isolated after lysis of cells in TRI Reagent according to the manufacturer's details. Isolated RNA was converted to cDNA using the GeneAmp RNA-PCR kit (PerkinElmer LAS GmbH, Juegesheim, Germany). cDNA was amplified using Real-Time quantitative PCR (RT-qPCR) on the Rotor-Gene Q in combination with the QuantiTect SYBR Green RT-PCR kit (Qiagen, Hilden, Germany). Amplification conditions were according to the manufacturer's protocol. As a reference gene GAPDH and $\beta$-actin were used. Levels of mRNA were quantified using Rotor-Gene series software v2.3.1 (Qiagen, Hilden, Germany). Primer pairs (Table I) were used at a final concentration of $0.8 \mu \mathrm{M}$. Cycling conditions involved $3 \mathrm{~min}$ at $95^{\circ} \mathrm{C}$ followed by 40 cycles of $1 \mathrm{~min}$ at $95^{\circ} \mathrm{C}, 1 \mathrm{~min}$ at $55^{\circ} \mathrm{C}$ and $2 \mathrm{~min}$ at $72^{\circ} \mathrm{C}$. Each RT-qPCR was performed in duplicate.

Sequencing of bisulfite-modified genomic DNA. Genomic DNA samples from normal and cancer cell lines were isolated using the Blood \& Cell Culture DNA Mini Kit (Qiagen, Hilden, Germany). DNA methylation was analysed using bisulfite-modified genomic DNA for a nested PCR amplification and subsequent bidirectional sequencing of the proximal part of the SERPINA5 promoter, ranging from $-193 \mathrm{bp}$ to $+56 \mathrm{bp}$ relative to the predicted TSS. Applied primer pairs are listed in Table I. The conditions for amplification were as follows: 15 cycles with extrinsic primers at $60-50^{\circ} \mathrm{C}$ as touch-down for 1 min followed by 35 cycles with intrinsic primers at $94^{\circ} \mathrm{C}$ for $45 \mathrm{sec}, 57^{\circ} \mathrm{C}$ for $45 \mathrm{sec}$ and $72^{\circ} \mathrm{C}$ for $1 \mathrm{~min}$. After checking the purity of amplified products by agarose gel electrophoresis, PCR products were sequenced using the $3730 \mathrm{XL}$ $\mathrm{ABI} /$ Hitachi Analyser. Sequences of bisulfite-treated genomic DNA were compared with those of untreated genomic DNA to verify the efficiency of bisulfite treatment. Ratios of cytosine/thymine and guanine/adenine residues in the forward and reverse sequence were respectively calculated to assess the extent of methylation at $8 \mathrm{CpG}$ sites (from 1 to 8 , according to the numbering used).

Methylation-specific high resolution melting analysis of bisulfitemodified DNA. Methylation-specific high resolution melting (MSHRM) analysis using SERPINA5 primers for bisulfite-modified DNA samples (Table I) was carried out to assess the extent of methylation of $\mathrm{CpG}$ sites from 1 to 5 in the selected region from -211 bp to -26 bp relative to the TSS of the SERPINA5 gene. The applied primer pairs were designed using MethPrimer software (26) and resulted in PCR products of $185 \mathrm{bp}$. Rotor-Gene Q and the EpiTect MS-HRM PCR kits were used according to the manufacturer's instructions (Qiagen, Hilden, Germany). Bisulfitemodified unmethylated and methylated standard DNAs (Qiagen, Hilden, Germany) were used for calibration. The applied primer pairs are shown in Table I. A standard curve with different DNA methylation ratios was calculated for each run.

Digital droplet PCR for the quantification of DNA methylation. All reagents, instruments and software used to study $\mathrm{CpG}$ methylation by means of droplet digital PCR (ddPCR) were purchased from Bio-Rad (Bio-Rad Laboratories GmbH, München, Germany). The ddPCR-reactions were set up by preparing a $20 \times-$ primer/probe mix from a $100 \mu \mathrm{M}$ primer stock and a $100 \mu \mathrm{M}$ probe stock. Primers and probes were used at a final concentration of $0.9 \mu \mathrm{M}$ and $0.25 \mu \mathrm{M}$, respectively. The reaction setup was performed in a final volume of $20 \mu \mathrm{l}$, of which $10 \mu \mathrm{l}$ were made up of the $2 x$ ddPCR Supermix, $1 \mu$ l of the aforementioned $20 x-$ primer/probe-mix, up to $3 \mu \mathrm{l}$ of the DNA template, and leftover volume was filled with RNase-/DNase-free water. 
Table I. Sequences of forward $(f)$ and reverse $(r)$ primers and probes.

\begin{tabular}{|c|c|c|}
\hline Gene & Primers ( $5^{\prime}$ to $\left.3^{\prime}\right)$ & Product size (bp) \\
\hline & RT-qPCR & \\
\hline \multirow[t]{2}{*}{ SERPINA5 } & (f) TCTTCCTCCTCTTGTGCCTG & \\
\hline & (r) TGCTCACAGGGGAGAAGAAG & 206 \\
\hline \multirow{2}{*}{$\beta$-Actin } & (f) CACCACACCTTCTACAATGAGC & \\
\hline & (r) CAGAGGCGTACAGGGATAGC & 178 \\
\hline \multirow[t]{3}{*}{ GAPDH } & (f) AAATCAAGTGGGGCGATGCTG & \\
\hline & (r) GCAGAGATGATGACCCTTTTG & 118 \\
\hline & Methylation analysis by sequencing of bisulfite modified DNA & \\
\hline \multirow[t]{2}{*}{ SERPINA5 (extrinsic) } & (f) TTGTTTTTGGAATGTTAATAATAGG & \\
\hline & (r) CACACAATAAAAATAACAATCCCT & 328 \\
\hline \multirow[t]{3}{*}{ SERPINA5 (intrinsic) } & (f) TGGTTTTGTTTTTTAGGAGTGTTTA & \\
\hline & (r) ACTAACTAAATTACAACCAACATT & 249 \\
\hline & MS-HRM methylation analysis of bisulfite modified DNA & \\
\hline \multirow{3}{*}{ SERPINA5 (1-5 CpGs) } & (f) AATAATAGGGAGGTTTTTTGGTTTT & \\
\hline & (r) СССТTCСТTТССТААСАААТTTC & 185 \\
\hline & dd-PCR methylation analysis of bisulfite modified DNA & \\
\hline \multirow[t]{2}{*}{ SERPINA5 } & (f) GGGAGTTGTGTTTGTAGGTAGG & \\
\hline & (r) AACCAACCCТАТСТCСАCCC & 101 \\
\hline Probe (unmethylated) & FAM-CCACCCCAAAACAACCCCTTCC-BHQ1 & \\
\hline Probe (methylated) & $\begin{array}{l}\text { HEX-CCGCCCCGAAACAACCCCTTCC-BHQ1 } \\
\text { (CpGs at }-19 \text { bp and }-14 \text { bp) }\end{array}$ & \\
\hline
\end{tabular}

The reaction mix was transferred strip-wise with a multipipette to a DG8 cartridge, and $70 \mu$ Droplet Generation Oil was being added to each well on the cartridge. The cartridge was then sealed with a DG8 gasket and put in the QX100 Droplet Generator, which generated up to 16,000 droplets in a volume of about $40 \mu \mathrm{l}$. These were then transferred to a PCR plate and heat sealed with the PX1 PCR Plate Sealer. The droplet emulsion was thermally cycled in the following conditions: denaturing at $95^{\circ} \mathrm{C}$ for $10 \mathrm{~min}, 40$ cycles of PCR at $94^{\circ} \mathrm{C}$ for $30 \mathrm{~s}$ and $57^{\circ} \mathrm{C}$ for $1 \mathrm{~min}$, and a final extension at $98^{\circ} \mathrm{C}$ for $10 \mathrm{~min}$. The annealing/extension step was performed at a temperature determined beforehand with a gradient ddPCR to ensure the best possible performance. After thermal cycling, the plate was loaded into the QX100 Droplet Reader and the droplet fluorescence was analysed with QuantaSoft V1.6.6.0320.

In silico analyses. Comparisons between SERPINA5 promoter methylation in cancer and adjacent non-cancer tissue probes were performed using the human pan-cancer methylation database MethHC (http://methhc.mbc.nctu.edu.tw/php/index.php). MethHC is a web based resource focused on DNA methylation of human cancers and uses integrated data such as DNA methylation, gene expression, microRNA methylation, microRNA expression, and the correlation of methylation and gene expression from the TCGA (The Cancer Genome Atlas) (27). Methprimer software (26) was used to map $\mathrm{CpG}$ islands in the promoter region of the SERPINA5 gene and for primer design. Potential binding sites for transcription factors were identified by the PROMO v.3 software (28).

Data analysis. Differences between groups were analysed with the Kruskal-Wallis one-way test of variance on ranks. The association of DNA methylation with SERPINA5 expression in different cells was assessed using the Spearman method for correlation. All statistical analyses were performed using the statistics module integrated in the SigmaPlot 11.2 software (Systat Software GmbH, Erkrath, Germany). Differences were considered significant at $p<0.05$.

\section{Results}

To investigate the DNA methylation patterns of the SERPINA5 promoter in different cancers, we firstly searched the MethHC database. The results showed a significant elevation of the methylation degree of SERPINA5 in tissue samples from prostate adenocarcinoma relative to adjacent non-tumor samples. Differences in methylation between tumor- and nontumor specimens were assessed by selecting the promoter (region defined as $0.5 \mathrm{~kb}$ upstream to $0.5 \mathrm{~kb}$ downstream of the transcription start site, TSS) and its region TSS-200 


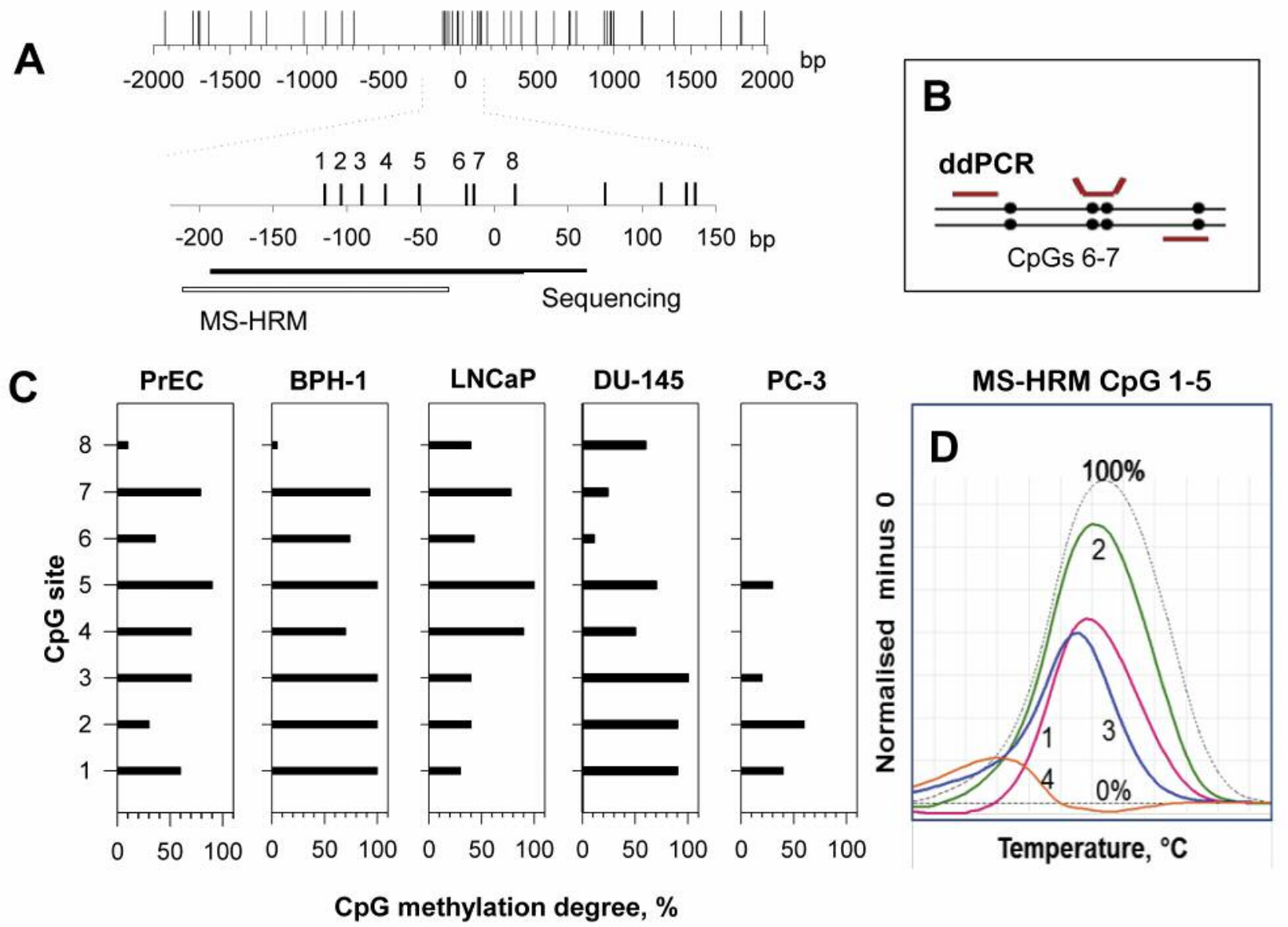

Figure 1. Differential methylation of CpG sites of the proximal promoter of the SERPINA5 gene in normal, benign hyperplastic and malignant prostate epithelial cells. (A): Graph illustrates the density of CpG sites 1-8 in the region from -2 $\mathrm{kb}$ upstream to $2 \mathrm{~kb}$ downstream of the transcription start site (TSS) of human SERPINA5 gene (Gene ID: 5104). TSS corresponds to the first nucleotide of cDNA encoding the SERPINA5-201 ENST00000329597 transcript (NCBI reference sequence: NM_000624.5). Horizontal bars mark the regions of the SERPINA5 promoter studied by DNA sequencing (black) and methylation-specific high resolution melting (open). (B) Schematic representation of primers (horizontal bars) and fluorescent probes (U-shaped figure) used by ddPCR analysis to study the methylation degree of CpG sites 6 and 7 of the SERPINA5 promoter. (C) Methylation values (\%) of CpG sites 1-8 of the SERPINA5 promoter in prostate cells determined by direct sequencing of bisulfite-modified genomic DNA. (D) MS-HRM difference plots of fluorescence signals of CpG sites 1-5 of the SERPINA5 promoter normalized to unmethylated standard DNA in PrEC (1), BPH-1 (2), LNCaP (3), and PC-3 (4) cells. Analyses were performed using standard DNA with methylation degrees of $0 \%$ and $100 \%$ (dotted lines).

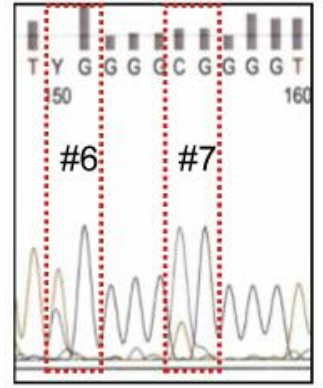

PrEC

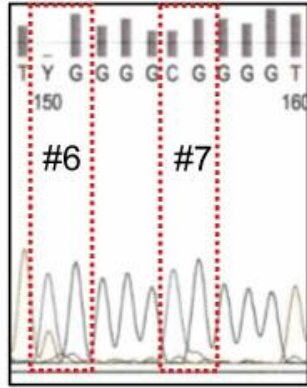

BPH-1

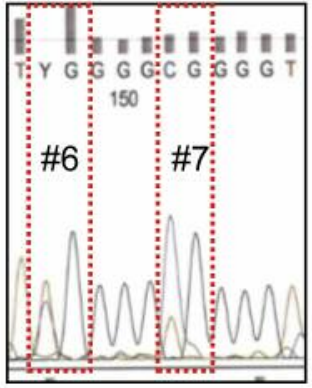

LNCaP

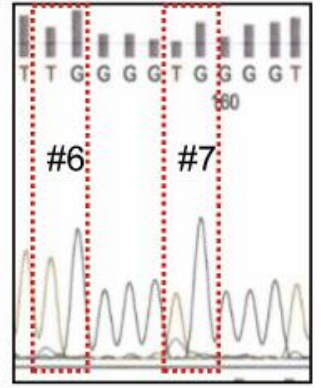

DU-145

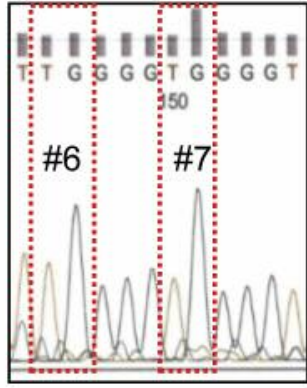

PC-3

Figure 2. Sequencing plots demonstrating differences in methylation degrees of CpG sites 6 and 7 of the SERPINA5 proximal promoter in prostate cells. Nucleotide pair TG indicates complete lack of methylation, CG complete methylation, and YG a mixed methylation status. 


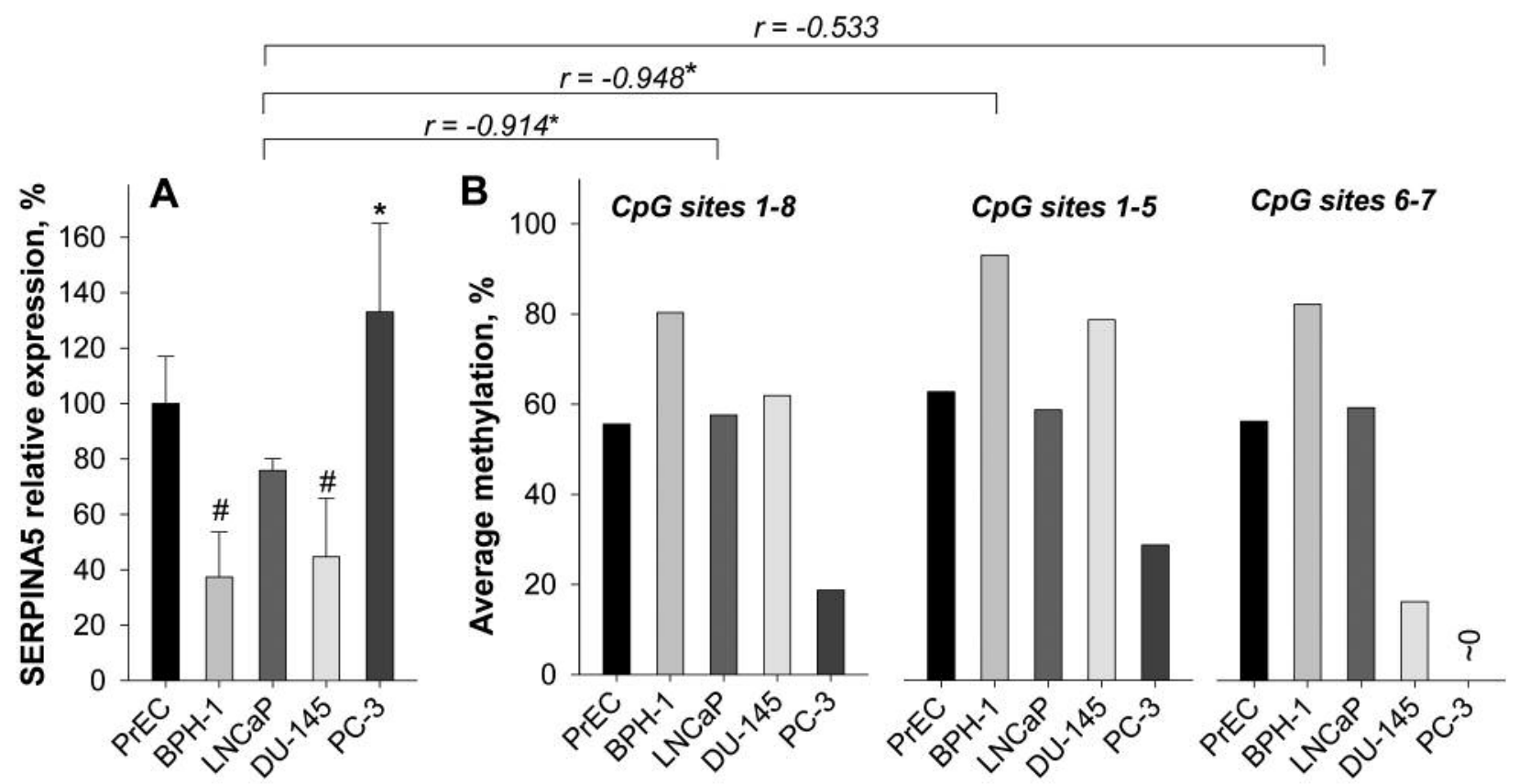

Figure 3. Relationship between SERPINA5 expression and level of average methylation of $C p G$ sites 1-5, 6 and 7, and 1-8 in normal, benign hyperplastic and malignant prostate epithelial cells. (A) SERPINA5 expression was measured using qPCR with GAPDH and $\beta$-actin as reference genes. Bars show the mean \pm SEM of three independent experiments in duplicates. Significance between expression values was analysed by multiple comparisons versus PrEC (Holm-Sidak method): ${ }^{*} p<0.05$ and ${ }^{\#} p<0.001$. (B) Average methylation values (\%) of different groups of CpG sites within the SERPINA5 promoter of PrEC, BPH-1, LNCaP, DU-145 and PC-3 cells determined by direct sequencing of bisulfite-modified genomic DNA. Values of the Pearson correlation coefficient ( $r$ ) between SERPINA5 expression and average methylation of $C p G$ sites 1-5, 6-7 and 1-8 are shown. ${ }^{*} p<0.05$.

(0.2 $\mathrm{kb}$ upstream of the TSS). In addition to prostate cancer, significant changes in the methylation degree of the SERPINA5 promoter were also observed in invasive breast carcinoma and thyroid carcinoma. Three methylation probes, namely cg00917847, cg13984563 and cg15509705, are commonly used to study methylation of the TSS-200 region of SERPINA5. SERPINA5 expression in cancer specimens inversely correlated with the methylation level of these $\mathrm{CpG}$ loci, wherein the values of the correlation coefficient markedly differed depending on cancer type and $\mathrm{CpG}$ location.

Analysis of $\mathrm{CpG}$ site distribution in the promoter region 2000 bp upstream of the TSS of the SERPINA5 gene revealed no $\mathrm{CpG}$ islands and only single $\mathrm{CpG}$ sites. A number of $\mathrm{CpGs}$ surrounding the transcription start site are organised in clusters, one of which consisting of eight $\mathrm{CpG}$ sites is located in the above mentioned region TSS-200 (Figure 1A). The positions of $\mathrm{CpG}$ sites relative to the TSS are listed in Table II. These $\mathrm{CpG}$ sites overlap with multiple putative binding sites of transcription factors, including specificity protein 1 (SP1) and 3 (SP3), activating enhancerbinding protein 2 -alpha (AP-2 $\alpha$ ), interferon regulatory factor 1 (IRF-1), c-Ets-1, p53 tumour suppressor protein, E2F-1 and others, as predicted by PROMO v.3 software.
Table II. Characteristics of CpG sites 1-8 of SERPINA5 proximal promoter.

\begin{tabular}{lccc}
\hline CpG site & $\begin{array}{c}\text { Location } \\
\text { relative TSS }\end{array}$ & $\mathrm{r}^{\mathrm{a}}$ & $\begin{array}{c}\text { ID of available } \\
\text { Illumina CG-probe }\end{array}$ \\
\hline 1 & -115 & -0.758 & $\operatorname{cg} 00917847$ \\
2 & -104 & -0.672 & \\
3 & -90 & -0.865 & \\
4 & -74 & -0.591 & $\operatorname{cg} 13984563$ \\
5 & -51 & -0.653 & \\
6 & -19 & -0.589 & $\operatorname{cg} 15509705$ \\
7 & -14 & -0.478 & \\
8 & 14 & -0.509 & \\
\hline
\end{tabular}

aPearson correlation (r) between SERPINA5 expression and CpG site methylation; bIllumina Infinium HM450 arrays containing probes for about 480,000 CpG dinucleotides located in functionally-relevant regions of the genome (31).

In the current study the degree of methylation of these eight $\mathrm{CpG}$ sites were analyzed in normal (PrEC), benign prostatic hyperplastic (BPH-1), and malignant (LNCaP, DU145, and PC-3) prostate epithelial cell lines. For this purpose, 

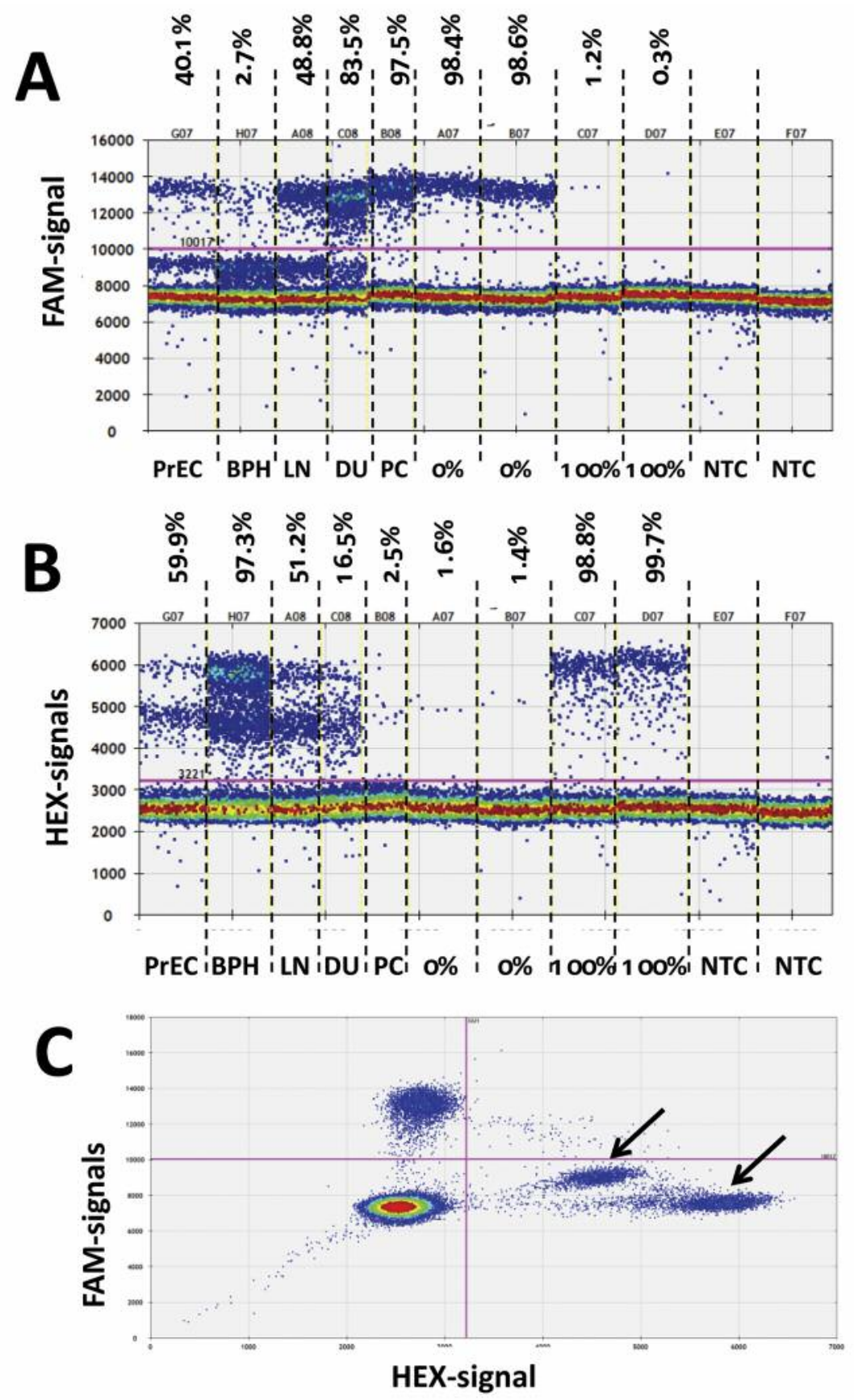

Figure 4. Analyses of the methylation status of CpG sites 6 and 7 of the SERPINA5 promoter in normal, benign prostatic hyperplastic cells and malignant prostate cell lines using ddPCR. $(A, B) d d P C R$ plots showing FAM and HEX signals for unmethylated $(A)$ and methylated $(B) C p G$ sites -19 and -14 in PrEC, BPH-1 (BPH), LNCaP (LN), PC-3 (PC), and DU-145 (DU) cells. 0\% and 100\% methylated DNA standards and non-template controls (NTC) are plotted as comparison. (C) 2D-plot analysis of data is shown. Arrows indicate homogeneously and heterogeneously methylated CpG sites -19 and -14 of the SERPINA5 promoter.

three different techniques, namely direct DNA sequencing (CpG sites 1-8), MS-HRM (CpG sites 1-5) and droplet digital PCR (CpG sites 6-7), were compared (Figure 1A, B). Direct sequencing of bisulfite-modified genomic DNA identified cell line specific patterns of SERPINA5 promoter methylation (Figure 1C). $\mathrm{CpG}$ sites from 1 to 5 were methylated in all prostate cells except $\mathrm{PC}-3$. Across all $\mathrm{CpG}$ cites the degree of methylation increased in the following order: PC-3 < DU-145 


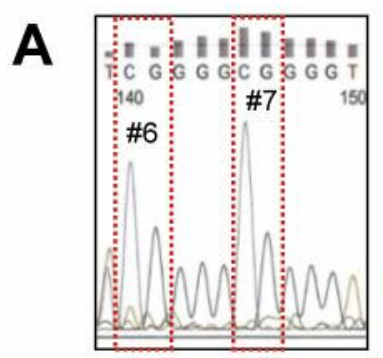

HMEC

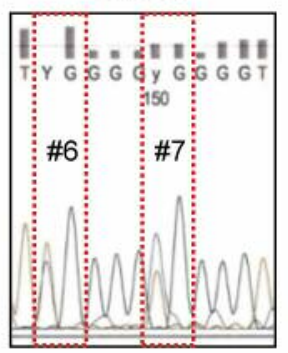

T-47D

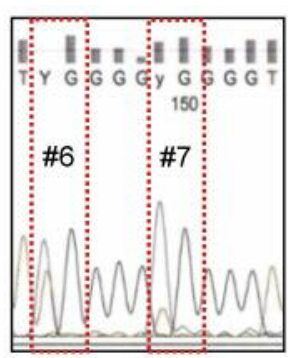

BT-474

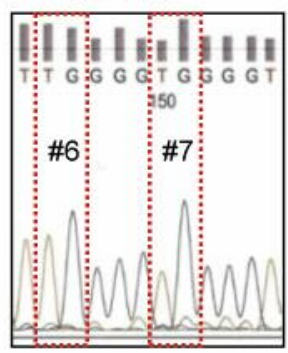

SK-BR-3

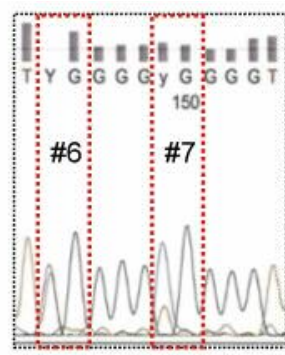

MDA-MB-453

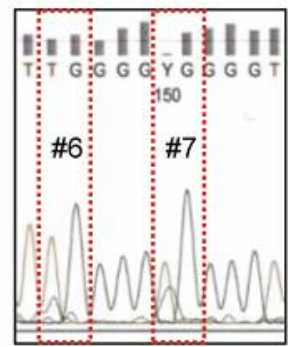

Cal-51

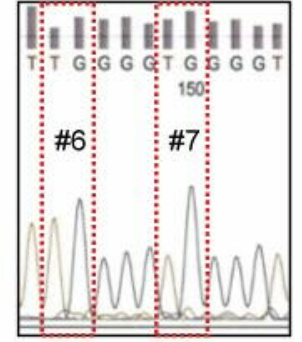

MCF-7

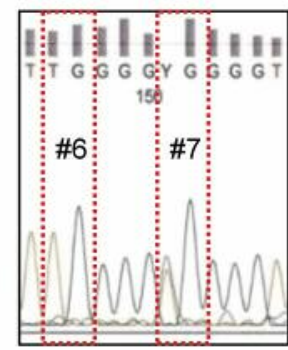

MDA-MB-231

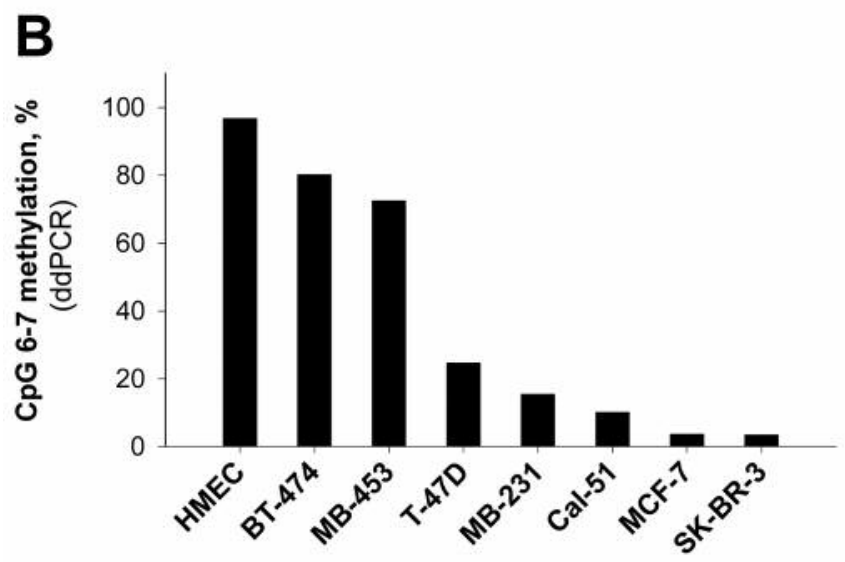

Figure 5. Methylation degree of CpG sites 6 and 7 of the SERPINA5 promoter in normal and malignant breast cells. (A) Sequencing plots show the methylation degree of $C p G$ sites 6 and 7 in normal and malignant breast cells. Nucleotide pair TG indicates complete lack of methylation, CG complete methylation, and YG a mixed methylation status. (B) Bars illustrate the methylation degree of CpG sites 6 and 7 measured by ddPCR of bisulfite-modified genomic DNA from normal and malignant breast cells.

$<\mathrm{LNCaP}=\mathrm{PrEC}<\mathrm{BPH}-1$ cells (Figure 1C), with the aggressive DU-145 and PC-3 malignant prostate cells being almost completely unmethylated at CpG sites -19 and -14 (\#6 and \#7). This was not the case for PrEC, BPH-1, and LNCaP cells (Figures $1 \mathrm{C}$ and 2).

The average methylation of $\mathrm{CpG}$ sites 1-5 was estimated by MS-HRM, confirming cell type-specific patterns of SERPINA5 promoter methylation. Data were consistent with those generated by direct sequencing, with high methylation of $\mathrm{CpG}$ sites 1-5 observed in BPH-1 cells and low methylation in PC-3 cells (Figure 1D).
According to relative quantification using RT-qPCR, levels of SERPINA5 mRNA were significantly down-regulated in BPH-1 and DU-145 cells in comparison to PrEC (Figure 2A). Transcript levels of SERPINA5 were significantly lower in LNCaP cells and moderately but not significantly higher in PC-3 relative to PrEC (Fiure 3A). Calculating the average methylation degree of $\mathrm{CpG}$ sites 1-8, 1-5 and 6-7, a strongly negative correlation between SERPINA5 expression and methylation of CpG sites 1-8 and 15 was observed in the analysed prostate cell lines (Figure 3B). Values of the Pearson correlation coefficient were lower $(\mathrm{r}=0.533)$ and non-significant for $\mathrm{CpG}$ sites 6-7. 
With the purpose of in-depth quantification of methylation of $\mathrm{CpG}$ sites -19 and -14 in non-cancerous and cancer cells with varying degrees of tumorigenic potential, droplet digital PCR using a probe selective for CpG sites -19 and -14 was applied (Figure 1B and Table I). Methylation degrees of these sites averaged to $16.5 \%$ and $2.5 \%$ in DU-145 and PC3 cells, respectively, whereas in BPH-1, PrEC and LNCaP cells the degree of methylation averaged to $97.3 \%, 59.9 \%$ and $51.2 \%$, respectively (Figure 4). Compared to MS-HRM, ddPCR-based $\mathrm{CpG}$ methylation analysis allowed the quantification of homogenously and heterogeneously methylated $\mathrm{CpG}$ alleles (Figure 4). For the degree of unmethylation only alleles with homogenously unmethylated CpG sites -19 and -14 were included (Figure 4A), whereas the methylated fraction comprised the homogenously ( $\mathrm{CpG}$ sites -19 and -14 were completely methylated) and heterogenously methylated alleles (either CpG site 6 or 7 was methylated) (Figure 4B). Importantly, ddPCR showed the best performance in distinguishing SERPINA5 promoter methylation between benign hyperplastic and malignant prostate cells among all applied techniques.

Since nearly complete unmethylation of CpG sites -19 and -14 was found in aggressive prostate cell lines, we also analysed the methylation of these $\mathrm{CpG}$ sites in mammary cancer cell lines differing in their molecular subtype and tumorigenic potential. Sequencing analysis identified different degrees of methylation across these cell types, similar to the data obtained in prostate cell lines (Figure 5A). Quantitative analyses using ddPCR demonstrated low methylation of $\mathrm{CpG}$ sites -19 and -14 in human metastatic breast carcinoma cell lines SK-BR-3 (3.4\%), MCF-7 (3.6\%), Cal-51 (10.1\%), MDA-MB-231 (15.4\%), and T-47D (24.6\%). In contrast, non-transformed HMEC, low tumorigenic cell line MDA-MB-453 and primary malignant cell line BT-474 were highly methylated with $95.8 \%, 72.5 \%$ and $80.2 \%$, respectively (Figure 5B).

\section{Discussion}

The present study focuses on the relationship between promoter methylation and expression of the SERPINA5 gene in normal (PrEC), benign hyperplastic (BPH-1) and malignant prostate cell lines (LNCaP, DU-145 and PC-3). The former was analysed by three different techniques, namely direct nucleotide sequencing, MS-HRM and ddPCR. For the first time, a strong demethylation of two $\mathrm{CpG}$ sites located at -19 and -14 bp upstream of the TSS in the SERPINA5 proximal promoter was shown in the aggressive cancer cell lines DU-145 and PC-3 in comparison to PrEC, $\mathrm{BPH}-1$, and LNCaP cells. In contrast to these unmethylated sites, the methylation degree of five additional $\mathrm{CpG}$ sites upstream of the TSS (1-5 according to our nomenclature in Figure 1) negatively and significantly correlated with the expression of SERPINA5 (Figure 3). This suggests that the role of promoter methylation in the regulation of SERPINA5 expression has to be considered and evaluated in the context of tumour type and that some $\mathrm{CpG}$ regions better reflect SERPINA5 expression than others. Our findings are consistent with the analysis of publicly available data sets from MethHC, in which the methylation degree of different regions of the SERPINA5 promoter negatively correlated with gene expression in tissue samples of prostate adenocarcinoma.

Epigenetic mechanisms such as DNA methylation, histone modification, and posttranscriptional gene regulations by non-coding RNAs play an important role in the control of gene expression (18). These mechanisms were detected at earlier stages of neoplastic transformation and considered as essential for cancer initiation and progression. Cellular DNA undergoes profound methylation changes during cancer development, with hypermethylation occurring in specific gene promoters often resulting in silencing of tumor suppressors and genes important for cellular growth, regulation and differentiation (20). According to the presented sequencing data, the studied CpG sites 1-8 of the SERPINA5 proximal promoter are differentially methylated in a cell type-dependent manner. Notably, CpG site 5 (-51 bp upstream of the TSS), corresponds to the Illumina Infinium HM450 array (21) CpG probe cg13984563, which was previously used as a methylation marker to study the epigenetic control of SERPINA5 expression in breast (14) and papillary thyroid cancer (21). Hypermethylation of two additional $\mathrm{CpG}$ sites of the SERPINA5 promoter, $113 \mathrm{bp}$ and $130 \mathrm{bp}$ downstream of the TSS, was also described in a study with papillary thyroid cancer (21). The methylation of these two $\mathrm{CpG}$ sites was not considered in the current study.

Amongst the here analysed non-transformed and malignant prostate cells, the strongest difference in the degree of methylation was observed in $\mathrm{CpG}$ sites -19 and 14. These two $\mathrm{CpG}$ sites were close to $100 \%$ methylated in BPH-1 cells and almost completely unmethylated in androgen-insensitive DU-145 and PC-3 cancer cells, representing a previously undescribed methylation signature of SERPINA5 (Figures 1 and 2). Interestingly, two of the studied breast cancer cell lines, Cal-51 and MDA-MB-231 belonging to aggressive basal molecular subtype, were also characterizsd by low methylation of CpG sites -19 and -14 of the SERPINA5 promoter (Figure 5). The applied methods to evaluate methylation of $\mathrm{CpG}$ sites -19 and -14 , namely sequencing and ddPCR, were consistent with each other, with ddPCR having a higher analytical sensitivity and accuracy enabling detection of rare unmethylated alleles as potential cancer risk biomarkers.

Several studies indicated a protective role of SERPINA5 against tumor development, invasiveness, and metastasis (3, 
$10,11,15,21)$. Elevated protein levels were detected in normal prostatic epithelia compared to tumor tissue, with a subpopulation of high-grade tumor cells having undetectable amounts of SERPINA5. These findings suggested that the loss of SERPINA5 in high-grade tumors may promote an invasive tumor growth pattern (23). SERPINA5 was also shown to regulate the invasive potential of renal cell carcinoma via inhibition of uPA secretion (13).

Based on the present study the question arises, to what extent data concerning decreased methylation of SERPINA5 in aggressive cancer cell lines can be related to human tissue samples, where higher SERPINA5 methylation was observed in primary prostate tumors compared to matched normal tissue. Cultured cell lines can be powerful model systems for the study of human tumorigenesis; however, the most intensively studied human prostate cancer cell lines, PC-3, DU-145, and LNCaP, were established from metastatic lesions, which are unlikely to accurately recapitulate the genetic composition and biological behaviour of primary prostate tumors (29). In this context, it would be of interest to study SERPINA5 levels in primary tumors and matched metastasis. In addition, the prostate gland is a multifaceted system, in which prostate epithelia and stroma have distinct physiological roles and gene expression profiles potentially leading to differential expression between monocultures of tumor cells and tissue samples comprised of several cell types (30).

In conclusion, in the current study a differential methylation of eight $\mathrm{CpG}$ sites of the SERPINA5 promoter was shown, from which five sites inversely correlated with gene expression. By using nucleotide sequencing, MSHRM and ddPCR analyses of bisulfite-modified DNA cellspecific patterns of DNA methylation were discovered, with distinct differences between BPH-1 cells and aggressive PC-3 and DU-145 prostate cancer cells especially at CpG sites 6 and 7 located at -19 and -14 bp upstream of the TSS. The newly established ddPCR-based high-performance assay for detection of methylation of CpG sites -19 and 14 is a useful tool for further studies quantifying methylation of the SERPINA5 promoter in human tumor and non-transformed tissue.

\section{Conflicts of Interest}

The Authors have no conflicts of interest to report.

\section{Acknowledgements}

The Authors are grateful to Dr. Sylke Winkler, Liane Funke and Dorit Pache for performing DNA sequencing with ABI 3.1 at the 3730 XL ABI Hitachi-sequencer (Max Planck Institute of Molecular Cell Biology and Genetics, DNA Sequencing Facility, Dresden) and to Margot Vogel and Romy Adler for their expert technical assistance.

\section{References}

1 Suzuki K: The multi-functional serpin, protein $\mathrm{C}$ inhibitor: beyond thrombosis and hemostasis. J Thromb Haemost 6: 20172026, 2008 .

2 Silverman GA, Bird PI, Carrell RW, Church FC, Coughlin PB, Gettins PG, Irving JA, Lomas DA, Luke CJ, Moyer RW, Pemberton PA, Remold-O'Donnell E, Salvesen GS, Travis J and Whisstock JC: The serpins are an expanding superfamily of structurally similar but functionally diverse proteins. Evolution, mechanism of inhibition, novel functions, and a revised nomenclature. J Biol Chem 276: 33293-33296, 2001.

3 Yang $\mathrm{H}$ and Geiger M: Cell penetrating SERPINA5 (Protein $\mathrm{C}$ inhibitor, PCI): more questions than answers. Semin Cell Dev Biol 62: 187-193, 2017.

4 Elisen MG, von dem Borne PA, Bouma BN and Meijers JC: Protein $\mathrm{C}$ inhibitor acts as a procoagulant by inhibiting the thrombomodulin-induced activation of protein $\mathrm{C}$ in human plasma. Blood 91: 1542-1547, 1998.

5 Espana F, Gilabert J, Estelles A, Romeu A, Aznar J and Cabo A: Functionally active protein $\mathrm{C}$ inhibitor/plasminogen activator inhibitor-3 (PCI/PAI-3) is secreted in seminal vesicles, occurs at high concentrations in human seminal plasma and complexes with prostate-specific antigen. Thromb Res 64: 309-320, 1991.

6 Francis RB Jr and Thomas W: Behaviour of protein C inhibitor in intravascular coagulation and liver disease. Thromb Haemost 52: $71-74,1984$

7 Sil H, Sen $\mathrm{T}$ and Chatterjee A: Fibronectin-integrin (alpha5beta1) modulates migration and invasion of murine melanoma cell line B16F10 by involving MMP-9. Oncol Res 19: 335-348, 2011.

8 Sieben NL, Oosting J, Flanagan AM, Prat J, Roemen GM, Kolkman-Uljee SM, van Eijk R, Cornelisse CJ, Fleuren GJ and van Engeland M: Differential gene expression in ovarian tumors reveals Dusp 4 and Serpina 5 as key regulators for benign behavior of serous borderline tumors. J Clin Oncol 23: 7257$7264,2005$.

9 Fortenberry YM, Brandal S, Bialas RC and Church FC: Protein $\mathrm{C}$ inhibitor regulates both cathepsin L activity and cell-mediated tumor cell migration. Biochim Biophys Acta 1800: 580-590, 2010.

10 Jing Y, Jia D, Wong CM, Oi-Lin Ng I, Zhang Z, Liu L, Wang Q, Zhao F, Li J, Yao M, Wu X and He X: SERPINA5 inhibits tumor cell migration by modulating the fibronectin-integrin $\beta 1$ signaling pathway in hepatocellular carcinoma. Mol Oncol 8 : 366-377, 2014

11 Bijsmans IT, Smits KM, de Graeff P, Wisman GB, van der Zee AG, Slangen BF, de Bruïne AP, van Engeland M, Sieben NL and van de Vijver KK: Loss of SerpinA5 protein expression is associated with advanced-stage serous ovarian tumors. Mod Pathol 24: 463-470, 2011.

12 Kristjansdottir B, Partheen K, Fung ET, Marcickiewicz J, Yip C, Brännström $\mathrm{M}$ and Sundfeldt $\mathrm{K}$ : Ovarian cyst fluid is a rich proteome resource for detection of new tumor biomarkers. Clinical Proteomics 9: 14, 2012.

13 Wakita T, Hayashi T, Nishioka J, Tamaru H, Akita N, Asanuma K, Kamada H, Gabazza EC, Ido M, Kawamura J and Suzuki K: Regulation of carcinoma cell invasion by protein $\mathrm{C}$ inhibitor whose expression is decreased in renal cell carcinoma. Int $\mathrm{J}$ Cancer 108: 516-523, 2004 
14 Conway K, Edmiston SN, May R, Kuan PF, Chu H, Bryant C, Tse CK, Swift-Scanlan T, Geradts J, Troester MA and Millikan RC: DNA methylation profiling in the Carolina Breast Cancer Study defines cancer subclasses differing in clinicopathologic characteristics and survival. Breast Cancer Res 16: 450, 2014.

15 Asanuma K, Yoshikawa T, Hayashi T, Akita N, Nakagawa N, Hamada Y, Nishioka J, Kamada H, Gabazza EC, Ido M, Uchida A and Suzuki K: Protein C inhibitor inhibits breast cancer cell growth, metastasis and angiogenesis independently of its protease inhibitory activity. Int J Cancer 121: 955-965, 2007.

16 Castelló R, Landete JM, España F, Vázquez C, Fuster C, Almenar SM, Ramón LA, Radtke KP and Estellés A: Expression of plasminogen activator inhibitors type 1 and type 3 and urokinase plasminogen activator protein and mRNA in breast cancer. Thromb Res 120: 753-762, 2007.

17 Herman JG and Baylin SB: Gene silencing in cancer in association with promoter hypermethylation. N Engl J Med 349: 2042-2054, 2003.

18 Toiyama Y, Okugawa Y and Goel A: DNA methylation and microRNA biomarkers for noninvasive detection of gastric and colorectal cancer. Biochem Biophys Res Commun 455: 43-57, 2014

19 Willard SS and Koochekpour S: Regulators of gene expression as biomarkers for prostate cancer. Am J Cancer Res 2: 620-657, 2012.

20 Heichman KA and Warren JD: DNA methylation biomarkers and their utility for solid cancer diagnostics. Clin Chem Lab Med 50: 1707-1721, 2012.

21 Lee EK, Chung KW, Yang SK, Park MJ, Min HS, Kim SW and Kang HS: DNA methylation of MAPK signal-inhibiting genes in papillary thyroid carcinoma. Anticancer Res 33: 4833-4839, 2013.

22 Gopalan S, Kasza A, Xu W, Kiss DL, Wilczynska KM, Rydel RE and Kordula T: Astrocyte- and hepatocyte-specific expression of genes from the distal serpin subcluster at 14q32.1 associates with tissue-specific chromatin structures. J Neurochem 94: 763-773, 2005.

23 Cao Y, Becker C, Lundwall A, Christensson A, Gadaleanu V, Lilja $\mathrm{H}$ and Bjartell A: Expression of protein $\mathrm{C}$ inhibitor (PCI) in benign and malignant prostatic tissues. Prostate 57: 196-204, 2003.
24 Glasscock LN, Rehault SM, Gregory CW, Cooper ST, Jackson TP, Hoffman $M$ and Church FC: Protein $C$ inhibitor (plasminogen activator inhibitor-3) expression in the CWR22 prostate cancer xenograft. Exp Mol Pathol 79: 23-32, 2005.

25 Menschikowski M, Hagelgans A, Gussakovsky E, Kostka H, Paley EL and Siegert G: Differential expression of secretory phospholipases A2 in normal and malignant prostate cell lines: regulation by cytokines, cell signaling pathways, and epigenetic mechanisms. Neoplasia 10: 279-286, 2008.

$26 \mathrm{Li}$ LC and Dahiya R: MethPrimer: designing primers for methylation PCRs. Bioinformatics 18: 1427-1431, 2002.

27 Huang WY, Hsu SD, Huang HY, Sun YM, Chou CH, Weng SL and Huang HD: MethHC: a database of DNA methylation and gene expression in human cancer. Nucleic Acids Res 43(Database issue): D856-861, 2015.

28 Farré D, Roset R, Huerta M, Adsuara JE, Roselló L, Albà MM and Messeguer X: Identification of patterns in biological sequences at the ALGGEN server: PROMO and MALGEN. Nucleic Acids Res 31: 3651-3653, 2003.

29 Schwab TS, Stewart T, Lehr J, Pienta KJ, Rhim JS and Macoska JA: Phenotypic characterization of immortalized normal and primary tumor-derived human prostate epithelial cell cultures. Prostate 44: 164-171, 2000.

30 Gregg JL, Brown KE, Mintz EM, Piontkivska H and Fraizer GC: Analysis of gene expression in prostate cancer epithelial and interstitial stromal cells using laser capture microdissection. BMC Cancer 10: 165, 2010.

31 Bibikova M, Barnes B, Tsan C, Ho V, Klotzle B, Le JM, Delano D, Zhang L, Schroth GP, Gunderson KL, Fan JB and Shen R: High density DNA methylation array with single $\mathrm{CpG}$ site resolution. Genomics 98: 288-295, 2011.

Received September 11, 2017

Revised September 29, 2017 Accepted October 2, 2017 